\title{
Posterior surgical approach procedures for cervical myelopathy
}

\author{
Pedro H.I. Pohl \\ ABC Medical School \\ Marcel Jun Tamaoki \\ Universidade Federal de São Paulo \\ Luciano M. Rodrigues \\ ABC Medical School \\ Kristen Radcliff \\ Thomas Jefferson University \\ Takashi Yurube \\ Kobe University Graduate School of Medicine
}

Follow this and additional works at: https://jdc.jefferson.edu/orthofp

See next page for additional authors

Part of the Orthopedics Commons

Let us know how access to this document benefits you

\section{Recommended Citation}

Pohl, Pedro H.I.; Tamaoki, Marcel Jun; Rodrigues, Luciano M.; Radcliff, Kristen; Yurube, Takashi; Kang, James D.; and Belloti, João Carlos, "Posterior surgical approach procedures for cervical myelopathy" (2015). Department of Orthopaedic Surgery Faculty Papers. Paper 107.

https://jdc.jefferson.edu/orthofp/107

This Article is brought to you for free and open access by the Jefferson Digital Commons. The Jefferson Digital Commons is a service of Thomas Jefferson University's Center for Teaching and Learning (CTL). The Commons is a showcase for Jefferson books and journals, peer-reviewed scholarly publications, unique historical collections from the University archives, and teaching tools. The Jefferson Digital Commons allows researchers and interested readers anywhere in the world to learn about and keep up to date with Jefferson scholarship. This article has been accepted for inclusion in Department of Orthopaedic Surgery Faculty Papers by an authorized administrator of the Jefferson Digital Commons. For more information, please contact: JeffersonDigitalCommons@jefferson.edu. 


\section{Authors}

Pedro H.I. Pohl, Marcel Jun Tamaoki, Luciano M. Rodrigues, Kristen Radcliff, Takashi Yurube, James D. Kang, and João Carlos Belloti 


\section{(F) Cochrane Library}

Cochrane Database of Systematic Reviews

\section{Posterior surgical approach procedures for cervical myelopathy (Protocol)}

Pohl PHI, Tamaoki MJ, Rodrigues LM, Radcliff KE, Yurube T, Kang JD, Belloti JC

Pohl PHI, Tamaoki MJ, Rodrigues LM, Radcliff KE, Yurube T, Kang JD, Belloti JC.

Posterior surgical approach procedures for cervical myelopathy.

Cochrane Database of Systematic Reviews 2015, Issue 3. Art. No.: CD011553.

DOI: 10.1002/14651858.CD011553.

www.cochranelibrary.com 
TABLE OF CONTENTS

HEADER . . . . . . . . . . . . . . . . . . . . . . . . . . . . . . . . . . . . 1

ABSTRACT . . . . . . . . . . . . . . . . . . . . . . . . . . . . . . . . . . . . . . . 1

BACKGROUND . . . . . . . . . . . . . . . . . . . . . . . . . . . . . . . . . . . .

OBJECTIVES . . . . . . . . . . . . . . . . . . . . . . . . . . . . . . . . . . . . . . .

METHODS . . . . . . . . . . . . . . . . . . . . . . . . . . . . . . . . . . . . . . . .

ACKNOWLEDGEMENTS . . . . . . . . . . . . . . . . . . . . . . . . . . . . . . . . . . . . . . . .

REFERENCES . . . . . . . . . . . . . . . . . . . . . . . . . . . . . . . . . . . . . . 6

APPENDICES . . . . . . . . . . . . . . . . . . . . . . . . . . . . . . . . . . . . . 9

CONTRIBUTIONS OF AUTHORS . . . . . . . . . . . . . . . . . . . . . . . . . . . . . . . . . . . . . . . . 14

DECLARATIONS OF INTEREST . . . . . . . . . . . . . . . . . . . . . . . . . . . . . . . . 14

SOURCES OF SUPPORT . . . . . . . . . . . . . . . . . . . . . . . . . . . . . . . . . . 


\title{
[Intervention Protocol] \\ Posterior surgical approach procedures for cervical myelopathy
}

Pedro HI Pohl ${ }^{1}$, Marcel Jun Tamaoki ${ }^{2}$, Luciano M Rodrigues ${ }^{1}$, Kristen E Radcliff ${ }^{3}$, Takashi Yurube ${ }^{4}$, James D Kang ${ }^{5}$, João Carlos Belloti $^{2}$

${ }^{1}$ Discipline of Orthopaedic Surgery and Traumatology / Spine Surgery Group, ABC Medical School, São Paulo, Brazil. ${ }^{2}$ Department of Orthopaedics and Traumatology, Universidade Federal de São Paulo, São Paulo, Brazil. ${ }^{3}$ Rothman Institute, Department of Orthopedic Surgery, Thomas Jefferson University, Egg Harbor, NJ, USA. ${ }^{4}$ Department of Orthopaedic Surgery, Kobe University Graduate School of Medicine, Kobe, Japan. ${ }^{5}$ Orthopaedic Department, University of Pittsburgh Medical Center, Pittsburgh, PA, USA

Contact address: Pedro HI Pohl, Discipline of Orthopaedic Surgery and Traumatology / Spine Surgery Group, ABC Medical School, 321 Dr. Henrique Calderazzo ST, 5th Floor, São Paulo, 09190-615, Brazil. pedropohl@live.com.

Editorial group: Cochrane Back and Neck Group.

Publication status and date: New, published in Issue 3, 2015.

Citation: Pohl PHI, Tamaoki MJ, Rodrigues LM, Radcliff KE, Yurube T, Kang JD, Belloti JC. Posterior surgical approach procedures for cervical myelopathy. Cochrane Database of Systematic Reviews 2015, Issue 3. Art. No.: CD011553. DOI: 10.1002/14651858.CD011553.

Copyright (C) 2015 The Cochrane Collaboration. Published by John Wiley \& Sons, Ltd.

\begin{abstract}
A B S T R A C T
This is the protocol for a review and there is no abstract. The objectives are as follows:

The main objective of this review is to assess the effects of laminectomy and fusion versus laminoplasty for multilevel cervical stenosis with myelopathy, on treatment outcomes such as pain, quality of life, functional and neurological improvement, and complication rates.
\end{abstract}

\section{B A C K G R O U N D}

\section{Description of the condition}

Cervical myelopathy is a clinical condition of spinal cord dysfunction which demonstrates characteristic findings of upper motor neuron syndrome, also referred to as central motor neuron disease. One of the initial symptoms of myelopathy often involves loss of finger dexterity in the upper extremities. Patients usually report progressive loss of function including difficulty with fine movements in ordinary activities such as buttoning buttons and handwriting. Some patients also present with lower limb spasticity and a resultant wide based, shuffling gait. Ultimately, this ataxia may become so severe that patients become wheelchair dependent. In addition to ataxia, the lower limbs often demonstrate hyperreflexia and pathologic reflexes. These pathologic reflexes result from a pathway defect between peripheral nerves and the brain modulation, including Hoffmann's (thumb and index finger interphalangeal joints flex when distal phalanx of third finger is flicked), Babinsky's (extensor plantar reflex) and Openheimer's (dorsiflexion of the hallux elicited by stimulation of middle third of tibia) reflexes (Clarke 1956; Payne 1957; Tracy 2010; Veidlinger 1981). The most common cause of cervical myelopathy is degenerative changes resulting in acquired stenosis. Cervical stenosis is a radiographic diagnosis based on the narrowing of the spinal canal, possibly leading to spinal cord compression and the clinical syndrome of cervical spondylotic myelopathy. The normal spinal canal diameter is 15 to $17 \mathrm{~mm}$, depending on measurement techniques and population. Patients with a cervical canal under $13 \mathrm{~mm}$ of anterior 
to posterior diameter are considered to have stenosis (Chazono 2012; Edwards 1983) and progression of cervical myelopathy has been observed to occur in patients with midsagittal diameter less than 12mm (Boden 1990). Axial sections demonstrating an area below $40 \mathrm{~mm}^{2}$ and compression ratio (relation between sagittal and transverse diameter) beneath $40 \%$ are also less favourable prognostic factors (Law 1993; Veidlinger 1981).

Degenerative changes, including hypertrophy of facet and uncovertebral joints (cartilage interface that connects adjacent vertebral bodies), osteophytosis (bone spurs), disc herniation, thickening of ligamentum flavum (ligaments that surround the posterior aspect of spinal cord) and ossification of posterior longitudinal ligament (structure related to anterior aspect of spinal cord), are the predominant structural changes that cause cervical stenosis (Bernhardt 1993; Edwards 2003).

Cervical spondylotic myelopathy can be classified by the syndromes it produces, divided into five categories:

1. Transverse lesion syndrome, where all spinal tracts are equally affected;

2. Motor system syndrome, where motor spinal tracts are involved, resulting in spasticity and loss of strength;

3. Central cord syndrome, where upper extremities are more severely affected than lower in sensory and motor aspects;

4. Brown-Séquard syndrome, where a patient presents ipsilateral motor deficits with contralateral sensory deficits; and

5. Braquialgia, where there is motor or sensory impairment in the upper extremity (Crandall 1966).

Cervical myelopathy also can be defined by the site in which compression occurs, separated into four syndromes:

1. Medial syndrome, with impairment primarily of long tract signs;

2. Lateral syndrome, characterized by radicular lesion;

3. Medial and lateral syndromes combined; and

4. Vascular syndrome, characterized by quickly progressive myelopathy signs due to vascular insufficiency (Ferguson 1985)

\section{Description of the intervention}

Posterior surgical decompression procedures for cervical myelopathy are often used in patients with three or more levels involved. The most commonly performed approaches are laminectomy alone, laminectomy with arthrodesis, and laminoplasty (Highsmith 2011; Lee 2007; Manzano 2012).

Cervical laminectomy is the oldest procedure described for decompression of the cervical spinal cord. It has been applied since the early 1900s. It consists of removing posterior elements of the spinal canal including the lamina, spinal processes, interspinous and supraspinous ligaments, ligamentum flavum, and sometimes, portions of the articular facets (Jenkins 1973; Nurick 1972; Veidlinger 1981).

Cervical laminectomy is commonly performed along with fusion to prevent the development of post laminectomy kyphotic defor- mity. The most commonly performed fusion method is arthrodesis of the facet joints and lateral masses with lateral mass and pedicle screws. Nevertheless some spine surgeons choose not to use arthrodesis, but rather release the spinal cord using laminectomy alone or the skip laminectomy (a variation of classical laminectomy with less bone removed) (Heller 2001; Manzano 2012; Shiraishi 2003).

Cervical laminoplasty is a canal-expanding procedure to preserve motion in the cervical spine. Hirabayashi described the expansive open-door laminoplasty procedure in the late 1970s. The key principle of this procedure is to minimize disruption of the posterior structures of cervical spine including ligaments and muscular insertions, keeping them relatively preserved. By using a drill on both sides of the posterior vertebra, both the internal and external cortex of the lamina on one side is cut while only the external cortex is cut on the opposite site, and then the lamina is opened like a door (Hirabayashi 1978; Hirabayashi 1983).

A variation of Hirabayashi technique is called French-door or double-door laminoplasty (Kaneyama 2010; Kurokawa 1982; Okada 2009). This procedure is performed by making a cut in the centre of the spinal process and the external cortex on both sides of the lamina. Some surgeons use suture wire or bone grafts to keep the lamina or spinous process open. However, there are other options to maintain the decompression, such as small plates, anchors or similar devices (Lee 2007; Mitsunaga 2012).

\section{How the intervention might work}

Decompression of cervical spinal stenosis using a posterior approach, such as laminectomy or laminoplasty techniques, increases the space in the cervical canal. These procedures decompress the spinal cord directly by removing compressive posterior structures, and/or indirectly by increasing the transverse diameter of the cervical canal when compression is due to anterior structures (Herkowitz 2011).

\section{Why it is important to do this review}

Myelopathy secondary to spondylotic cervical canal stenosis is currently the most common cause of cervical spinal disease observed in patients over 55 years. The natural history of untreated cervical myelopathy is stepwise progression towards severe disability. As such, surgical decompression with or without fusion is the treatment of choice for patients with symptomatic cervical myelopathy (Bernhardt 1993; Edwards 2003; Fehlings 2013a; Fehlings 2013b; Lees 1963; Sampath 2000).

Posterior approaches such as laminectomy with bone fusion and laminoplasty are techniques most applied in patients with three or more compressive levels without kyphotic deformity. Laminectomy, as previously described, consists of removing vertebral lamina, spinal process, and ligaments to promote a wide decompres- 
sion. After decompression a rigid cervical fusion is performed with rods and screws. Laminoplasty also increases the cervical canal diameter but with less anatomical disruption. Additionally, cervical fusion is not performed. Since the spine is not fused and anatomical structures are preserved, it is argued that cervical spines after laminoplasty will maintain a greater range of motion when compared to cervical spines after laminectomy and fusion. Despite this theoretical advantage, clinically detectable differences between these two treatments have yet to be demonstrated (Bartels 2007; Hirabayashi 1983; Kaneyama 2010; Koakutsu 2010; Manzano 2012; Okada 2009; Shiraishi 2003; Woods 2011; Yukawa 2007). The best surgical option for treating patients with multilevel cervical myelopathy remains a subject of great controversy. A recent survey of North American spine surgeons evaluated the most common surgical treatments employed for multilevel cervical spinal stenosis (three or more compressed levels), without kyphotic deformity, and with myelopathic symptoms. Findings indicate that laminectomy with fusion is the procedure of choice for $70 \%$ of surgeons, laminoplasty for $23 \%$, and that just $7 \%$ of respondents chose laminectomy without fusion to treat this specific patient population (Manzano 2012).

One of the major concerns of spine surgeons who perform multilevel cervical decompression is the development of postoperative cervical kyphosis (forward curvature). Cervical kyphosis may lead to a deterioration in neurological state due to compression of the spinal cord over the ventral kyphotic deformity. The normal cervical alignment averages $14.4^{\circ}$ of lordosis (Albert 1998).

Cervical laminectomy without fusion has an incidence of postoperative kyphosis ranging from 14\% to 47\% (Anderson 2009; Kato 1998; Ryken 2009; van Geest 2013). Rates of deterioration of neurological state associated with development of kyphosis can vary between 10\% and 39\% (Arnold 1993; Ebersold 1995; McAllister 2012; Yonenobu 1986). It is still uncertain to what extent cervical spine instability with consequent kyphosis contributes to late neurological deterioration. These data may explain why cervical laminectomy without fusion is no longer a popular procedure. Laminectomy with fusion and laminoplasty seem to be the most commonly chosen procedures by spine surgeons due to safety and ethical concerns. This Cochrane systematic review will be conducted in order to identify randomized trials, quasi-randomized trials and non-randomized studies evaluating each procedure, and to clarify which is most beneficial to patients with cervical stenosis. In conclusion, since cervical myelopathy affects a considerable portion of the population and due to the lack of consensus regarding treatment, this review will help to elucidate the clinical nuances of the most commonly performed posterior approach procedures in order to achieve wide cervical decompression. If successful, this review can change the perspective of treatment of cervical myelopathy, increase patients' quality of life, and optimize costs, providing evidence-based data in order to assist spine surgeons to treat multilevel cervical myelopathy worldwide.

\section{O B J E C T I VES}

The main objective of this review is to assess the effects of laminectomy and fusion versus laminoplasty for multilevel cervical stenosis with myelopathy, on treatment outcomes such as pain, quality of life, functional and neurological improvement, and complication rates.

\section{METHODS}

\section{Criteria for considering studies for this review}

\section{Types of studies}

We will include randomized controlled trials (RCTs) in this review. Moreover, since the preliminary search showed few RCTs on this topic, we will also include quasi-randomized controlled trials (qRCTs) (i.e., non-random method of allocation such as hospital record number, date of care) as well as prospective observational studies with a control group, referred to here as non-randomized studies (NRSs).

\section{Types of participants}

Participants in included studies must meet the following criteria:

1. Older than 18 years;

2. Presence of symptomatic cervical myelopathy secondary to multilevel cervical canal stenosis (three or more levels of impairment);

3. Associated spondylosis, disc herniation, or ossification of the posterior longitudinal ligament (OPLL);

4. Magnetic resonance imaging (MRI) of cervical spinal cord compression.

We will exclude studies involving participants who present with the following:

1. Any degree of kyphotic deformity on cervical spine;

2. Previous surgical intervention.

\section{Types of interventions}

We will include studies that compare laminectomy with fusion to laminoplasty, as these are the most commonly applied posterior approach procedures (Manzano 2012) for patients with multilevel cervical stenosis.

We will also compare different laminoplasty surgeries such as single-door or double-door techniques as a secondary goal.

Studies that compare laminectomy without fusion to other interventions will be excluded. 


\section{Types of outcome measures}

Primary and secondary outcomes may be measured at different time points. We define short-term follow-up as closest to four weeks and long-term follow-up as closest to one year (Furlan 2009). Outcomes related to neurological function, quality of life, pain, range of motion and reoperation rates may be measured at short-term and/or long-term follow-up. However we believe that long-term measurements will provide better indications of the patient's status after treatment, with the exception of nerve root palsy (usually a transitory symptom, C5 is the most commonly affected root), which is an important short-term outcome. Besides primary and secondary outcomes, major (e.g. mortality, irreversible paralysis, deep infection) and minor (e.g. superficial wound infection, dysphagia) adverse events also will be evaluated and included in the Discussion (Fehlings 2012).

\section{Primary outcomes}

- Measurements of neurological function based on a validated specific cervical scale:

- Japanese Orthopaedic Association (JOA) Scale (JOA

1976; JOA 1994)

- Modified Japanese Orthopaedic Association (mJOA)

Scale (Benzel 1991; Chiles 1999)

- Neck Disability Index (Vernon 1991)

- Nurick Scale (Nurick 1972)

- Health related quality of life analysis will be measured through validated quality of life scales (e.g. Short-Form 36, JOA Cervical Myelopathy Evaluation Questionnaire/JOACEMQ, EuroQol) (Ware 1992, Nikaido 2009, EuroQol 1990)

- Pain (visual analogue scale) (Revill 1976)

- Cervical range of motion

- Kyphosis incidence (the cervical sagittal alignment has been demonstrated to be an important outcome in spine surgery procedures. Previous studies demonstrated that laminectomy as well as laminoplasty techniques can be followed by the development of kyphotic deformity, a situation that can lead ultimately to an important deterioration of neurological status)

\section{Secondary outcomes}

- Nerve root palsy and recovery rates (most commonly affected is C5 nerve root)

- Reoperation rates

\section{Search methods for identification of studies}

\section{Electronic searches}

We will search the following databases:
1. Cochrane Central Register of Controlled Trials (CENTRAL; The Cochrane Library)

2. Cochrane Back Group Trials Register (through CENTRAL, and through the Cochrane Register of Studies (CRS), for studies not in CENTRAL)

3. MEDLINE (OvidSP) and MEDLINE Non-Indexed and In-Process Citations (OvidSP)

4. EMBASE (OvidSP)

5. PubMed, for studies not in MEDLINE

6. Latin-American and Caribbean Center on Health Sciencies Information (LILACS)

No restrictions as to language or date of publication will be applied, and all databases will be searched from the date of their inception to present. We will use the search methods recommended by Furlan 2009 and Higgins 2011 and the strategies developed by the Cochrane Back Review Group (CBRG). A draft strategy for MEDLINE can be found in Appendix 1. It will be translated as closely as possible across the other databases.

We will also search the following trial registries for ongoing studies:

1. WHO International Clinical Trials Registry Platform ( WHO ICTRP)

2. United States National Institute of Health registry of clinical trials (ClinicalTrials.gov)

3. Biomed Central's Database of Current Controlled Trials ( ISRCTN registry)

\section{Searching other resources}

Personal communication with experts in this field.

We will also check reference lists of relevant review articles and trials.

\section{Data collection and analysis}

\section{Selection of studies}

Two authors (PHIP and TY) will analyse studies identified in the search. These authors will independently apply the inclusion criteria to select the potentially relevant studies by screening titles and abstracts as well as full texts. In case of disagreement, they will consult a third author (MJST) who will evaluate the full texts only.

\section{Data extraction and management}

All relevant data will be extracted independently by two authors (PHIP and MJST) and entered into a data sheet, in pairs, including information such as trial authors, affiliation, conflicts of interest, study design, number of patients, population characteristics, brief description of surgical techniques, follow-up periods, complications, reoperation data, outcome data, and a brief summary 
of risk of bias. The authors will enter all data into Review Manager 5.3 (RevMan 2014).

\section{Assessment of risk of bias in included studies}

Two authors, PHIP and MJST, will evaluate each study independently. Included studies will be assessed using the 'Risk of bias' criteria recommended by the CBRG (Furlan 2009; Higgins 2011) associated with items from the Downs and Black checklist (Downs 1998). In cases of disagreement, a third author (LMRR) will be consulted. These criteria are allocated into five bias categories, namely selection bias, performance bias, attrition bias, detection bias and selective outcome reporting, with a total of 13 distinct items (Appendix 2).

We will evaluate the studies according the criteria mentioned above where each item will be scored according to its characteristics as 'high', 'low' or 'unclear' and reported in the 'Risk of bias' table. If one or more criteria are rated as 'unclear', we will contact the study authors for additional information. The overall risk of bias of each one of the five bias categories will be rated as 'bias' or 'no bias'.

For grading the evidence quality we will assess studies as follows: the evidence level from studies that we qualified as 'no bias' for all five bias categories will not be downgraded. For studies that have three or fewer categories qualified as having 'bias', evidence will be downgraded by -1 point. Studies that have four or five categories qualified as having 'bias' will be downgraded by -2 points.

We are aware of several potential confounders when examining treatment effects in this area. Some of them include age (patients less than 60 years old at the time of surgery have better neurological recovery), cervical curvature magnitude (normal preoperative cervical lordosis can be responsible for better outcome results), symptom duration (patients with longer symptomatology tend to have worse recovery), transverse area of spinal cord on MRI, etiology of cervical spinal stenosis (different populations susceptible to cervical spondylosis versus OPLL may have different outcomes), and differences related to follow-up outcome forms (Kohno 1997; Morio 2001; Naderi 1998; Tetreault 2013; Yamazaki 2003).

\section{Measures of treatment effect}

We will calculate risk ratios (RRs) with a 95\% confidence interval for dichotomous outcomes. However, for outcomes which are measured using different scales, we will report a standardized mean difference (SMD). For continuous outcomes we will calculate mean differences (MDs) comparing different studies with different weights according to the standard deviation (SD) also using a $95 \%$ confidence interval. When more than two interventions are present in same study, we will calculate a single 'pairwise' comparison to avoid 'double-counting' of patients.

\section{Unit of analysis issues}

The data of patients reported in included studies will be used as the unit of analysis.

\section{Dealing with missing data}

In studies where relevant data are missing, we will contact the authors of trials for additional information. Studies that have more than $20 \%$ of data missing will be subject to a sensitivity analysis to evaluate the impact of excluding these data from the analysis.

\section{Assessment of heterogeneity}

The $\mathrm{I}^{2}$ statistic and $\mathrm{Chi}^{2}$ test will be used to assess the clinically homogenous studies, while heterogenousstudies will be evaluated qualitatively. Then the review team will use clinical judgment to examine the potential sources of heterogeneity, such as differences in interventions, characteristics of participants, interventions, controls groups, and outcomes. If heterogeneity can be explained, we will present the results of each subgroup separately (Furlan 2009).

\section{Assessment of reporting biases}

To minimize the risk of publication bias, we will search trial registry databases for ongoing and unpublished trials.

Duplicate publication bias is related to use of the same patients' data in multiple publications. To reduce the risk of bias, all such studies should be combined and analysed as a single study. Moreover to avoid language reporting bias, no limitations of language will be placed on the initial searches.

\section{Data synthesis}

If possible, the results will be pooled. We will calculate the risk ratios (RRs) with a $95 \%$ confidence interval for dichotomous outcomes. If included studies show clinical and methodological diversity, the random-effects model will be applied. The $\mathrm{Chi}^{2}$ test will be used to assess heterogeneity with results inferior to 0.1 indicating significant statistical difference.

If a meta-analysis is not possible, the results from clinically comparable trials will be described qualitatively in the text.

Regardless of whether there are sufficient data available to use quantitative analysis to summarize the data, we will assess the overall quality of the evidence for each outcome. To accomplish this, we will use the GRADE approach, as recommended in the Cochrane Handbook for Systematic Reviews of interventions (Higgins 2011) and adapted in the updated CBRG method guidelines (Furlan 2009). Factors that may decrease the quality of the evidence are: study design and risk of bias, inconsistency of results, indirectness, imprecision (sparse data) and other factors (e.g. reporting bias). The quality of the evidence for a specific outcome will be reduced by a level, according to the performance of the studies against these five factors. 
High quality evidence: there are consistent findings among at least $75 \%$ of RCTs with low risk of bias, consistent, direct and precise data and no known or suspected publication biases. Further research is unlikely to change either the estimate or our confidence in the results.

Moderate quality evidence: one of the domains is not met. Further research is likely to have an important impact on our confidence in the estimate of effect and may change the estimate.

Low quality evidence: two of the domains are not met. Further research is very likely to have an important impact on our confidence in the estimate of effect and is likely to change the estimate. Very low quality evidence: three of the domains are not met. We are very uncertain about the results.

No evidence: no RCTs were identified that addressed this outcome.

\section{'Summary of findings' tables}

The 'Summary of findings' tables will include the main comparison of this review, laminectomy and fusion versus laminoplasty. In order to assess the treatments we will report the following outcomes in each table:

1. Neurological function improvement;

2. Quality of life analysis;

3. Pain evaluation;

4. Cervical range of motion;

5. Kyphosis incidence;

6. C5 root palsy;

7. Reoperation rates.

\section{Subgroup analysis and investigation of heterogeneity}

We aim to perform subgroup analyses in order to explore heterogeneity due to the following factors:

1. Surgical technique applied, comparing different

laminoplasty techniques

2. Patient characteristics:

- Physiopathology of cervical stenosis (development, degenerative, OPLL)

- Degree of neurological deficit

- Levels affected

- Age

- Sagittal alignment before surgical procedure

\section{Sensitivity analysis}

A sensitivity analysis will be performed to investigate the effects of any important sources of bias.

A glossary of relevant terms can be found in Appendix 3.

\section{ACKNOW LEDGEMENTS}

The authors would like to acknowledge:

- The Cochrane Back Group for continuous encouragement and support provided to produce this study.

- The authors' home institutions.

- All professionals involved in this project, in particular Tiffany Dugan, Karl Henrikson and Sergio Tanaka.

- The patience and love of our families.

\section{R E F E R E N C E S}

\section{Additional references}

\section{Albert 1998}

Albert TJ, Vacarro A. Postlaminectomy kyphosis. Spine 1998;23(24):2738-45.

Anderson 2009

Anderson PA, Matz PG, Groff MW, Heary RF, Holly LT, Kaiser MG, et al. Joint Section on Disorders of the Spine and Peripheral Nerves of the American Association of Neurological Surgeons and Congress of Neurological Surgeons. Laminectomy and fusion for the treatment of cervical degenerative myelopathy. Journal of Neurosurgery. Spine 2009;11(2):150-6.

Arnold 1993

Arnold H, Feldmann U, Missler U. Chronic spondylogenic cervical myelopathy. A critical evaluation of surgical treatment after early and long-term follow-up. Neurosurgical Review 1993;16(2):105-9.

\section{Bartels 2007}

Bartels RH, Verbeek AL, Grotenhuis JA. Design of Lamifuse: a randomised, multi-centre controlled trial comparing laminectomy without or with dorsal fusion for cervical myeloradiculopathy. BMC Musculoskeletal Disorders 2007;8:111.

\section{Benzel 1991}

Benzel EC, Lancon J, Kesterson L, Hadden T. Cervical laminectomy and dentate ligament section for cervical spondylotic myelopathy. Journal of Spinal Disorders 1991;4 (3):286-95.

Bernhardt 1993

Bernhardt M, Hynes RA, Blume HW, White AA 3rd. Cervical spondylotic myelopathy. Journal of Bone and Joint 
Surgery. American Volume 1993;75(1):119-28.

\section{Boden 1990}

Boden SD, McCowin PR, Davis DO, Dina TS, Mark AS, Wiesel S. Abnormal magnetic-resonance scans of the cervical spine in asymptomatic subjects. A prospective investigation. Journal of Bone and Joint Surgery. American Volume 1990;72(8):1178-84.

Chazono 2012

Chazono M, Tanaka T, Kumagae Y, Sai T, Marumo $\mathrm{K}$. Ethnic differences in pedicle and bony spinal canal dimensions calculated from computed tomography of the cervical spine: a review of the English-language literature. European Spine Journal 2012;21(8):1451-8.

Chiles 1999

Chiles BW 3rd, Leonard MA, Choudhri HF, Cooper PR. Cervical spondylotic myelopathy: patterns of neurological deficit and recovery after anterior cervical decompression. Neurosurgery 1999;44(4):762-9.

\section{Clarke 1956}

Clarke E, Robinson PK. Cervical myelopathy: a complication of cervical spondylosis. Brain 1956;79(3): 483-510.

\section{Crandall 1966}

Crandall PH, Batzdorf U. Cervical spondylotic myelopathy. Journal of Neurosurgery 1966;25(1):57-66.

Downs 1998

Downs S, Black N. The feasibility of creating a checklist for the assessment of the methodological quality both of randomised and non-randomised studies of health care interventions. Journal of Epidemiology and Community Health 1998;52:377-84.

\section{Ebersold 1995}

Ebersold MJ, Pare MC, Quast LM. Surgical treatment for cervical spondylitic myelopathy. Journal of Neurosurgery 1995;82(5):745-51.

\section{Edwards 1983}

Edwards WC, LaRocca H. The developmental segmental sagittal diameter of the cervical spinal canal in patients with cervical spondylosis. Spine 1983;8(1):20-7.

\section{Edwards 2003}

Edwards CC 2nd, Riew KD, Anderson PA, Hilibrand AS, Vaccaro AF. Cervical myelopathy. Current diagnostic and treatment strategies. Spine Journal 2003;3(1):68-81.

\section{EuroQol 1990}

EuroQol Group. EuroQol--a new facility for the measurement of health-related quality of life. Health Policy 1990;16(3):199-208.

\section{Fehlings 2012}

Fehlings MG, Smith JS, Kopjar B, Arnold PM, Yoon ST, Vaccaro AR, et al. Perioperative and delayed complications associated with the surgical treatment of cervical spondylotic myelopathy based on 302 patients from the AOSpine North America Cervical Spondylotic Myelopathy Study. Journal of Neurosurgery. Spine 2012;16(5):425-32.
Fehlings 2013a

Fehlings MG, Tetreault LA, Wilson JR, Skelly AC. Cervical spondylotic myelopathy: current state of the art and future directions. Spine 2013;38(22 Suppl 1):S1-8.

\section{Fehlings 2013b}

Fehlings MG, Wilson JR, Kopjar B, Yoon ST, Arnold PM, Massicotte EM, et al. Efficacy and safety of surgical decompression in patients with cervical spondylotic myelopathy: results of the AOSpine North America prospective multi-center study. Journal of Bone and Joint Surgery. American Volume 2013;95(18):1651-8.

\section{Ferguson 1985}

Ferguson RJ, Caplan LR. Cervical spondylitic myelopathy. Neurologic Clinics 1985;3(2):373-82.

\section{Furlan 2009}

Furlan AD, Pennick V, Bombardier C, van Tulder M, Editorial Board Cochrane Back Review Group. 2009 Updated method guidelines for systematic reviews in the Cochrane Back Review Group. Spine 2009;34(18): 1929-41.

\section{Heller 2001}

Heller JG, Edwards CC 2nd, Murakami H, Rodts GE. Laminoplasty versus laminectomy and fusion for multilevel cervical myelopathy: an independent matched cohort analysis. Spine 2001;26(12):1330-6.

\section{Herkowitz 2011}

Herkowitz HN, Rothman RH, Simeone FA. RothmanSimeone, The Spine. 6th Edition. Philadelphia: Saunders Elsevier, 2011.

\section{Higgins 2011}

Higgins JPT, Green S (editors). Cochrane Handbook for Systematic Reviews of Interventions. The Cochrane Collaboration 2011, Version 5.0.1 [updated March 2011]. Available from www.cochrane-handbook.org.

Highsmith 2011

Highsmith JM, Dhall SS, Haid RW Jr, Rodts GE Jr, Mummaneni PV. Treatment of cervical stenotic myelopathy: a cost and outcome comparison of laminoplasty versus laminectomy and lateral mass fusion. Journal of Neurosurgery. Spine 2011;14(5):619-25.

Hirabayashi 1978

Hirabayashi K. Expansive open-door laminoplasty for cervical spondylotic myelopathy. Japanese Journal of Surgery 1978;32:1959-63.

Hirabayashi 1983

Hirabayashi K, Watanabe K, Wakano K, Suzuki N, Satomi K, Ishii Y. Expansive open-door laminoplasty for cervical spinal stenotic myelopathy. Spine 1983;8(7):693-9.

\section{Jenkins 1973}

Jenkins DH. Extensive cervical laminectomy. Long-term results. British Journal of Surgery 1973;60(11):852-4.

\section{JOA 1976}

Japanese Orthopaedic Association. Japanese Orthopaedic Association scoring system for cervical spondylotic 
myelopathy. Nippon Seikeigeka Gakkai Zasshi 1976;50: 18-19.

JOA 1994

Japanese Orthopaedic Association. Japanese Orthopaedic Association scoring system for cervical myelopathy (172 version and 100 version) [in Japanese with English translation]. Nippon Seikeigeka Gakkai Zasshi 1994;68: 490-503.

\section{Kaneyama 2010}

Kaneyama S, Sumi M, Kanatani T, Kasahara K, Kanemura A, Takabatake M, et al. Prospective study and multivariate analysis of the incidence of $\mathrm{C} 5$ palsy after cervical laminoplasty. Spine 2010;35(26):E1553-8.

\section{Kato 1998}

Kato Y, Iwasaki M, Fuji T, Yonenobu K, Ochi T. Long-term follow-up results of laminectomy for cervical myelopathy caused by ossification of the posterior longitudinal ligament. Journal of Neurosurgery 1998;89(2):217-23.

\section{Koakutsu 2010}

Koakutsu T, Morozumi N, Ishii Y, Kasama F, Sato T, Tanaka Y, et al. Anterior decompression and fusion versus laminoplasty for cervical myelopathy caused by soft disc herniation: a prospective multicenter study. Journal of Orthopaedic Science 2010;15(1):71-8.

\section{Kohno 1997}

Kohno K, Kumon Y, Oka Y, Matsui S, Ohue S, Sakaki $S$. Evaluation of prognostic factors following expansive laminoplasty for cervical spinal stenotic myelopathy. Surgical Neurology 1997;48(3):237-45.

\section{Kurokawa 1982}

Kurokawa T, Tsuyama N, Tanaka H, Kobayashi M, Machida H, Izuka T, et al. Enlargement of spinal canal by the sagittal splitting of the spinous process. Bessatsu Seikeigeka 1982;2:234-40.

Law 1993

Law MD Jr, Bernhardt M, White AA 3rd. Cervical spondylotic myelopathy: a review of surgical indications and decision making. Yale Journal of Biology and Medicine 1993;66(3):165-77.

Lee 2007

Lee JY, Hanks SE, Oxner W, Tannoury C, Donaldson WF 3rd, Kang JD. Use of small suture anchors in cervical laminoplasty to maintain canal expansion: a technical note. Journal of Spinal Disorders \& Techniques 2007;20(1):33-5.

Lees 1963

Lees F, Turner JW. Natural history and prognosis of cervical spondylosis. British Medical Journal 1963;2(5373): 1607-10.

\section{Manzano 2012}

Manzano GR, Casella G, Wang MY, Vanni S, Levi AD. A prospective, randomized trial comparing expansile cervical laminoplasty and cervical laminectomy and fusion for multilevel cervical myelopathy. Neurosurgery 2012;70(2): 264-77.

\section{McAllister 2012}

McAllister BD, Rebholz BJ, Wang JC. Is posterior fusion necessary with laminectomy in the cervical spine?. Surgical Neurology International 2012;3(Suppl 3):S225-31.

\section{Mitsunaga 2012}

Mitsunaga LK, Klineberg EO, Gupta MC. Laminoplasty techniques for the treatment of multilevel cervical stenosis. Advances in Orthopedics 2012;2012:N/A. [DOI: http:// dx.doi.org/10.1155/2012/307916]

\section{Morio 2001}

Morio Y, Teshima R, Nagashima H, Nawata K, Yamasaki D, Nanjo Y. Correlation between operative outcomes of cervical compression myelopathy and mri of the spinal cord. Spine 2001;26(11):1238-45.

\section{Naderi 1998}

Naderi S, Ozgen S, Pamir MN, Ozek MM, Erzen C. Cervical spondylotic myelopathy: surgical results and factors affecting prognosis. Neurosurgery 1998;43(1):43-9; discussion 49-50.

\section{Nikaido 2009}

Nikaido T, Kikuchi S, Yabuki S, Otani K, Konno S. Surgical treatment assessment using the Japanese orthopedic association cervical myelopathy evaluation questionnaire in patients with cervical myelopathy: a new outcome measure for cervical myelopathy. Spine (Phila Pa 1976) 2009;34 (23):2568-72.

\section{Nurick 1972}

Nurick S. The natural history and the results of surgical treatment of the spinal cord disorder associated with cervical spondylosis. Brain 1972;95(1):101-8.

\section{Okada 2009}

Okada M, Minamide A, Endo T, Yoshida M, Kawakami M, Ando $\mathrm{M}$, et al. A prospective randomized study of clinical outcomes in patients with cervical compressive myelopathy treated with open-door or French-door laminoplasty. Spine 2009;34(11):1119-26.

\section{Payne 1957}

Payne EE, Spillane JD. The cervical spine; an anatomicopathological study of 70 specimens (using a special technique) with particular reference to the problem of cervical spondylosis. Brain 1957;80(4):571-96.

Revill 1976

Revill SI, Robinson JO, Rosen M, Hogg MI. The reliability of a linear analogue for evaluating pain. Anaesthesia 1976; 31(9):1191-8.

\section{RevMan 2014 [Computer program]}

The Nordic Cochrane Centre, The Cochrane Collaboration. Review Manager (RevMan). Version 5.3. Copenhagen: The Nordic Cochrane Centre, The Cochrane Collaboration, 2014.

Ryken 2009

Ryken TC, Heary RF, Matz PG, Anderson PA, Groff MW, Holly LT, et al. Joint Section on Disorders of the Spine and Peripheral Nerves of the American Association of Neurological Surgeons and Congress of Neurological 
Surgeons. Cervical laminectomy for the treatment of cervical degenerative myelopathy. Journal of Neurosurgery. Spine 2009;11(2):142-9.

\section{Sampath 2000}

Sampath P, Bendebba M, Davis JD, Ducker TB. Outcome of patients treated for cervical myelopathy. A prospective, multicenter study with independent clinical review. Spine 2000;25(6):670-6.

\section{Shiraishi 2003}

Shiraishi T, Fukuda K, Yato Y, Nakamura M, Ikegami T. Results of skip laminectomy-minimum 2-year follow-up study compared with open-door laminoplasty. Spine 2003; 28(24):2667-72.

\section{Tetreault 2013}

Tetreault LA, Kopjar B, Vaccaro A, Yoon ST, Arnold PM, Massicotte EM, et al. A clinical prediction model to determine outcomes in patients with cervical spondylotic myelopathy undergoing surgical treatment: data from the prospective, multi-center AOSpine North America study. Journal of Bone and Joint Surgery. American Volume 2013;95 (18):1659-66.

Tracy 2010

Tracy JA, Bartleson JD. Cervical spondylotic myelopathy. Neurologist 2010;16(3):176-87.

van Geest 2013

van Geest S, de Vormer AM, Arts MP, Peul WC, VleggeertLankamp CL. Long-term follow-up of clinical and radiological outcome after cervical laminectomy. European Spine Journal 2013; [Epub:ahead of print]. [DOI: 10.1007/ s00586-013-3089-6]

van Tulder 2003

van Tulder M, Furlan A, Bombardier C, Bouter L, Editorial Board Cochrane Back Review Group. Updated method guidelines for systematic reviews in the Cochrane Collaboration Back Review Group. Spine 2003;28(12): 1290-9.

\section{Veidlinger 1981}

Veidlinger OF, Colwill JC, Smyth HS, Turner D. Cervical myelopathy and its relationship to cervical stenosis. Spine $1981 ; \mathbf{6}(6): 550-2$.

\section{Vernon 1991}

Vernon H, Mior S. The Neck Disability Index: a study of reliability and validity. Journal of Manipulative and Physiological Therapeutics 1991;14(7):409-15.

Ware 1992

Ware JE Jr, Sherbourne CD. The MOS 36-item short-form health survey (SF-36). I. Conceptual framework and item selection. Medical Care 1992;30(6):473-83.

\section{Woods 2011}

Woods BI, Hohl J, Lee J, Donaldson W 3rd, Kang J. Laminoplasty versus laminectomy and fusion for multilevel cervical spondylotic myelopathy. Clinical Orthopaedics and Related Research 2011;469(3):688-95.

\section{Yamazaki 2003}

Yamazaki T, Yanaka K, Sato H, Uemura K, Tsukada A, Nose T. Cervical spondylotic myelopathy: surgical results and factors affecting outcome with special reference to age differences. Neurosurgery 2003;52(1):122-6; discussion 126.

\section{Yonenobu 1986}

Yonenobu K, Okada K, Fuji T, Fujiwara K, Yamashita $\mathrm{K}$, Ono K. Causes of neurologic deterioration following surgical treatment of cervical myelopathy. Spine 1986;11 (8):818-23.

\section{Yukawa 2007}

Yukawa Y, Kato F, Ito K, Horie Y, Hida T, Ito Z, Matsuyama Y. Laminoplasty and skip laminectomy for cervical compressive myelopathy: range of motion, postoperative neck pain, and surgical outcomes in a randomized prospective study. Spine 2007;32(18):1980-5.

* Indicates the major publication for the study

\section{A P PEN DICES}

\section{Appendix I. Search strategies}

\section{MEDLINE}

1. randomized controlled trial.pt.

2. controlled clinical trial.pt.

3. pragmatic clinical trial.pt.

4. comparative study.pt.

5. randomi\#ted.ti,ab.

6. placebo.ti,ab. 
7. drug therapy.fs.

8. randomly.ab,ti.

9. trial.ab,ti.

10. groups.ab.

11. exp Cohort Studies/

12. cohort\$.ti,ab.

13. comparative $\$$.ti,ab.

14. prospective $\$ . t i, a b$.

15. or/1-14

16. (animals not (humans and animals)).sh.

17. 15 not 16

18. (cervical adj5 myelopathy).ti,ab.

19. (cervical adj5 myeloradiculopathy).ti,ab.

20. (cervical adj5 radiculomyelopathy).ti,ab.

21. cervical vertebrae/

22. (cervical adj5 (compress $\$$ or stenosis or herniat $\$$ or degenerat $\$$ or radicul $\$$ or decompress $\$$ )).ti,ab.

23. longitudinal ligaments/

24. ossification of posterior longitudinal ligament/

25. ossification of posterior longitudinal ligament.ti,ab.

26. (cervical adj5 (lamin\#plasty or laminectomy or laminotomy)).ti,ab.

27. spinal osteophytosis/

28. spinal cord compression/

29. spondylosis/

30. spinal diseases/

31. limit 30 to $\mathrm{yr}=1963-1965$

32. or/18-29,31

33. laminectomy.mp.

34. lamin\#plasty.mp.

35. laminectomy/

36. laminoplasty/

37. laminotomy.mp.

38. ((dorsal or poster\$) adj5 (approach\$ or surg\$ or decompress\$ or instrument $\$$ or technique $\$)$ ).mp. [mp=title, abstract, original title, name of substance word, subject heading word, keyword heading word, protocol supplementary concept word, rare disease supplementary concept word, unique identifier]

39. decompression, surgical/

40. or/33-39

41. 17 and 32 and 40

\section{Appendix 2. Criteria for assessing risk of bias for internal validity for randomized and non- randomized studies (Furlan 2009; Downs and Black 1998)}

\section{Selection bias}

\section{Random sequence generation}

\section{Selection bias (biased allocation to interventions) due to inadequate generation of a randomised sequence}

There is a low risk of selection bias if the investigators describe a random component in the sequence generation process such as: referring to a random number table, using a computer random number generator, coin tossing, shuffling cards or envelopes, throwing dice, 
drawing of lots, minimization (minimization may be implemented without a random element, and this is considered to be equivalent to being random).

There is a high risk of selection bias if the investigators describe a non-random component in the sequence generation process, such as: sequence generated by odd or even date of birth, date (or day) of admission, hospital or clinic record number; or allocation by judgement of the clinician, preference of the participant, results of a laboratory test or a series of tests, or availability of the intervention. For non-randomized studies (NRSs) this will be rated as 'high bias' automatically.

\section{Allocation concealment}

\section{Selection bias (biased allocation to interventions) due to inadequate concealment of allocations prior to assignment}

There is a low risk of selection bias if the participants and investigators enroling participants could not foresee assignment because one of the following, or an equivalent method, was used to conceal allocation: central allocation (including telephone, web-based and pharmacy-controlled randomization); sequentially numbered drug containers of identical appearance; or sequentially numbered, opaque, sealed envelopes.

There is a high risk of bias if participants or investigators enroling participants could possibly foresee assignments and thus introduce selection bias, such as allocation based on: using an open random allocation schedule (e.g. a list of random numbers); assignment envelopes were used without appropriate safeguards (e.g. if envelopes were unsealed or non-opaque or not sequentially numbered); alternation or rotation; date of birth; case record number; or other explicitly unconcealed procedures.

In cases of NRSs this will be rated as high bias.

\section{Population*}

There is a low risk of bias if the participants included in different intervention groups are originating from the same population group.

\section{Timing*}

The risk of bias is considered low if the participants included in different intervention groups are recruited over the same time.

\section{Adjustment for confounding*}

There is a low risk of bias if there are no significant differences between the studied groups. Risk is considered high if there are no investigation or final analyses adjustment of the main confounders identified.

\section{Performance bias}

\section{Blinding of participants}

There is a low risk of performance bias if blinding of participants was ensured and it was unlikely that the blinding could have been broken; or if there was no blinding or incomplete blinding, but the review authors judge that the outcome is not likely to be influenced by lack of blinding.

\section{Blinding of personnel/care providers}

There is a low risk of performance bias if blinding of personnel was ensured and it was unlikely that the blinding could have been broken; or if there was no blinding or incomplete blinding, but the review authors judge that the outcome is not likely to be influenced by lack of blinding.

Posterior surgical approach procedures for cervical myelopathy (Protocol)

Copyright ( 2015 The Cochrane Collaboration. Published by John Wiley \& Sons, Ltd. 


\section{Compliance (adherence)}

There is low risk of bias if compliance with the interventions was acceptable, based on the reported intensity/dosage, duration, number and frequency for both the index and control intervention(s). For single-session interventions (e.g. surgery), this item is irrelevant (van Tulder 2003).

\section{Co-interventions}

There is low risk of bias if there were no co-interventions or they were similar between the index and control groups (van Tulder 2003).

\section{Attrition bias}

\section{Incomplete outcome data}

There is a low risk of attrition bias if there were no missing outcome data; reasons for missing outcome data were unlikely to be related to the true outcome (for survival data, censoring unlikely to be introducing bias); missing outcome data were balanced in numbers, with similar reasons for missing data across groups; for dichotomous outcome data, the proportion of missing outcomes compared with the observed event risk was not enough to have a clinically relevant impact on the intervention effect estimate; for continuous outcome data, the plausible effect size (difference in means or standardized difference in means) among missing outcomes was not enough to have a clinically relevant impact on observed effect size, or missing data were imputed using appropriate methods (if dropouts are very large, imputation using even 'acceptable' methods may still suggest a high risk of bias) (van Tulder 2003). The percentage of withdrawals and drop-outs should not exceed $20 \%$ for short-term follow-up and $30 \%$ for long-term follow-up and should not lead to substantial bias (these percentages are commonly used but arbitrary, not supported by literature) (van Tulder 2003).

\section{Intention-to-treat analysis}

There is low risk of bias if all randomized patients were reported/analyzed in the group to which they were allocated by randomization.

\section{Measurement/detection}

\section{Blinding of outcome assessment}

There is low risk of bias if blinding of the outcome assessment was ensured and it was unlikely that the blinding could have been broken; or if no blinding of incomplete blinding was performed, but the review authors judge that the outcome is not likely to be influenced by lack of blinding, or:

- for participant-reported outcomes in which the participant was the outcome assessor (e.g. pain, disability): risk of bias for outcome assessors is low if risk of bias for participant blinding is low;

- for outcome criteria that are clinical or therapeutic events that will be determined by the interaction between participants and care providers (e.g. co-interventions, length of hospitalization, treatment failure), in which the care provider is the outcome assessor: risk of bias for outcome assessors is low if risk of bias for care providers is low;

- for outcome criteria that are assessed from data from medical forms: risk of bias is low if the treatment or adverse effects of the treatment could not be noticed in the extracted data.

\section{Timing of outcome assessment}

There is low risk of bias if all important outcome assessments for all intervention groups were measured at the same time (van Tulder 2003), or if analyses adjust for different lengths of follow-up.

Posterior surgical approach procedures for cervical myelopathy (Protocol)

Copyright @ 2015 The Cochrane Collaboration. Published by John Wiley \& Sons, Ltd. 


\section{Selective reporting}

\section{Data dredging}

There is low risk of bias if all analyses were planned at the outset of the study, and high risk of bias if analyses were conducted retrospectively (e.g. retrospective unplanned subgroup analyses).

\section{Outcome measures}

There is low risk of reporting bias if the study protocol is available and all of the study's pre-specified (primary and secondary) outcomes that are of interest in the review have been reported in the pre-specified way, or if the study protocol is not available but it is clear that the published reports include all expected outcomes, including those that were pre-specified (convincing text of this nature may be uncommon).

There is a high risk of reporting bias if not all of the study's pre-specified primary outcomes have been reported; one or more primary outcomes is reported using measurements, analysis methods or subsets of the data (e.g. subscales) that were not pre-specified; one or more reported primary outcomes were not pre-specified (unless clear justification for their reporting is provided, such as an unexpected adverse effect); one or more outcomes of interest in the review are reported incompletely so that they cannot be entered in a metaanalysis; the study report fails to include results for a key outcome that would be expected to have been reported for such a study.

* Items are relevant to non-randomized studies exclusively.

\section{Appendix 3. Glossary}

Articular facets: Facet joint. Portion that connects vertebrae of different levels.

Arthrodesis: Surgical procedure to achieve bone fusion.

Arthroplasty: Replacement of a joint by an artificial device with the function of maintaining movement.

Ataxia: Loss of muscular coordination as a result of damage to the central nervous system.

Axial section: Cross section.

C5 root palsy: Special type of paralysis that affects the 5th cervical nerve root. Can recover spontaneously.

Cervical: Neck, upper spine.

Corpectomy: Surgical procedure in which the vertebral body is removed.

Cortex: Peripheral portion of an organ (e.g. bone cortex, cerebral cortex).

Discectomy: Surgical procedure of intervertebral disc removal.

Dorsiflexion of the hallux: Movement in dorsal direction of big toe, lift the big toe.

Dysphagia: Difficulty swallowing.

Hyperreflexia: Exaggerated reflexes.

Hypertrophy: A non-tumorous enlargement of an organ or a tissue.

Interphalangeal: Between phalanges (fingers and toes).

Interspinous and supraspinous ligaments: Posterior ligaments of the spine. They help to stabilize the vertebrae.

Ipsilateral: Located on the same side of the body.

Kyphosis: Curvature of the spine with anterior convexity. Normal for middle spine, pathological for cervical (upper) or lumbar (low) spine.

Lamina: Portion of posterior vertebra bone arch removed at laminectomy surgical procedure.

Lordosis: Curvature of the spine with posterior convexity. Normal for cervical (upper) and lumbar (low) spine.

Midsagittal: A plane dividing the human body in bilaterally symmetrical right and left halves.

Necrosis: Death of tissue due to injury or disease.

Ossification: Bone formation.

Pedicle: Bone structure responsible for connecting the vertebral body to posterior bone elements, also location of insertion of pedicle screws.

Pedicle screw: Screw inserted into vertebral pedicle.

Phalanx: Bone segment that compose fingers and toes.

Plantar: Of, relating to, or occurring on the sole of the foot.

Radicular: Related to spinal roots.

Posterior surgical approach procedures for cervical myelopathy (Protocol)

Copyright @ 2015 The Cochrane Collaboration. Published by John Wiley \& Sons, Ltd. 
Spinal cord: The main neuronal structure that conducts the stimulus for movement from the brain to the limbs as well as conducts the sensitive signals from the periphery to the brain.

Spinal process: Bony projection off the midline of posterior arch of each vertebra.

Spondylotic: Degenerative spinal change.

Upper motor neuron syndrome: Conjunct of symptoms associated with a lesion of motor neurons in the brain or spinal cord region. Vascular: Of, relating to, affecting, or consisting of a vessel or vessels.

\section{CONTRIBUTIONS OFAUTHORS}

Pedro Henrique Isoldi Pohl: Protocol, selection of the studies, data extraction analysis, clinical interpretation, methodological evaluation, RevMan data entry.

Marcel Jun Tamaoki: Protocol, selection of the studies, data extraction analysis, methodological evaluation, draft review.

Luciano Miller Reis Rodrigues: Protocol, data extraction, clinical interpretation

Kristen Emmanuel Radcliff: Protocol, clinical interpretation, draft review.

Takashi Yourube: Protocol, selection of the studies, clinical interpretation, draft review.

James D Kang: Protocol review, clinical interpretation, draft review.

João Carlos Belloti: Protocol review, methodological evaluation, draft review.

\section{DECLARATIONSOF INTEREST}

The authors declare that they have no conflicts of interest related to the subject of this systematic review.

\section{SOURCES OF SUPPORT}

\section{Internal sources}

- ABC Medical School, Brazil.

Support in the form of salary to PHIP and LMRR

- Universidade Federal de São Paulo, Brazil.

Support in the form of salary to MJT and JCB

- Thomas Jefferson University, USA.

Support in the form of salary to KER

- Kobe University Graduate School of Medicine, Japan.

Support in the form of salary to TY

- University of Pittsburgh Medical Center, USA.

Support in the form of salary to JDK 


\section{External sources}

- No sources of support supplied 